\title{
Humoral factors in serum of rats with chronic heart failure induce cardiomyocyte hypertrophy and reduce viability
}

\author{
Authors: Sarah El Rifai, ${ }^{A}$ Amalia Sintou, ${ }^{B}$ Catherine Mansfield, ${ }^{B}$ Charles Houston, ${ }^{B}$ Rasheda A Chowdhury, ${ }^{B}$ \\ Julia Gorelik ${ }^{B}$ and Susanne Sattler ${ }^{B}$
}

\section{Introduction}

Myocardial infarction (MI) is one of the leading causes of global mortality. Over the last decade, acute MI mortality has significantly decreased; however, fatal heart failure remains a common complication for post-MI survivors. ${ }^{1}$ Lack of oxygen due to MI causes cardiac tissue necrosis and, as a result, the induction of a pro-inflammatory environment. The necrotic tissue is replaced with a fibrotic scar rather than new cardiomyocytes. Excessive inflammation can result in increased death of cardiomyocytes and increased fibrosis, which reduces cardiac function. ${ }^{2}$ To optimise cardiac function, the heart may undergo hypertrophy. Although initially the hypertrophic response is advantageous, sustained hypertrophy is a contributor to heart failure. ${ }^{3}$

Here, we assess whether rodent chronic heart failure (CHF) serum contributes to the induction of cardiomyocyte hypertrophy and cell death.

\section{Materials and methods}

MI was induced by the surgical ligation of the left anterior descending (LAD) artery in adult male Sprague Dawley rats. Serum was collected from healthy controls, and 2 weeks (acute) and 16 weeks (CHF) post-MI.

Primary adult cardiomyocytes were isolated from healthy rats and treated with control and CHF serum for 24 and 48 hours for apoptosis and cytotoxicity assays.

Serum from healthy and CHF rats was further used to stimulate the HL-1.6 cardiomyocyte cell line for quantitative PCR (qPCR) expression analysis of $\alpha$-myosin heavy chain (MYH6) gene, and the hypertrophic genes $\beta$-myosin heavy chain (MYH7), atrial natriuretic factor (NPPA) and brain natriuretic peptide $(N P P B)$.

\section{Results and discussion}

CHF serum applied to healthy adult cardiomyocytes for $24 \mathrm{~h}$ resulted in increased apoptosis compared to stimulation with healthy serum (25.91 \pm 4.53 (mean \pm standard error of the mean; control; $n=5) ; 53.13 \pm 3.961(\mathrm{CHF} ; \mathrm{n}=10) ; \mathrm{p}=0.0025)$. CHF

Authors: ${ }^{\mathrm{A}}$ Imperial College London, London, UK; ${ }^{\mathrm{B}}$ National Heart and Lung Institute, London, UK serum stimulation for $24 \mathrm{~h}$ and $48 \mathrm{~h}$ showed reduced viability due to increased cytotoxicity compared to stimulation with healthy serum (day 1: 83.75 \pm 0.9287 (control; $n=5$ ); $73.45 \pm 2.722$ $(\mathrm{CHF}, \mathrm{n}=7) ; \mathrm{p}=0.0178$; day $2: 86.66 \pm 1.294$ (control; $\mathrm{n}=5)$; $73.43 \pm 3.508$ (CHF; $n=7), p=0.0066$ ).

The increase in apoptosis and cell death of cardiomyocytes after CHF serum stimulation suggests the presence of humoral factors in $\mathrm{CHF}$ serum that cause direct damage to the cardiomyocytes.

To assess the effect of CHF on cardiac hypertrophy, CHF serum was applied to a cardiomyocyte cell line (HL-1.6). This did not cause a significant change in the expression of NPPA, NPPB and MYH6 compared to control serum. However, CHF serum induced a significant increase in $M Y H 7$ expression compared to control (fold change $0.88 \pm 0.08$ (control; $\mathrm{n}=5$ ); $1.2 \pm 0.05$ (CHF; $\mathrm{n}=8$ ); $\mathrm{p}=0.0061$ ). Importantly, the $M Y H 7 /$ MYH6 ratio also increased (fold change 2.59 \pm 0.58 (control; $\mathrm{n}=5) ; 4.19 \pm 0.49(\mathrm{CHF} ; \mathrm{n}=8) ; \mathrm{p}=0.0322)$.

A previous study suggests that the increase in $\mathrm{MYH7}$ alone is sufficient to cause cardiac deterioration. ${ }^{4}$ Furthermore, it has been proposed that the shift from MYH6 to MYH7 expression might play a role in cardiac disease progression. ${ }^{4}$ Thus, the increase in MYH7 expression and MYH7/MYH6 ratio indicates a hypertrophic response.

\section{Conclusion}

These findings suggest that humoral factors present in CHF serum may be involved in the induction of the cellular processes leading to persistent myocardial damage and pathological cardiac hypertrophy.

\section{Conflicts of interest}

None declared.

\section{References}

1 Torabi A, Cleland JG, Rigby AS, Sherwi N. Development and course of heart failure after a myocardial infarction in younger and older people. J Geriatr Cardiol 2014;11:1-12.

2 Frangogiannis NG. Regulation of the inflammatory response in cardiac repair. Circ Res 2012;110:159-73.

3 Dirkx E, da Costa Martins PA, De Windt LJ. Regulation of fetal gene expression in heart failure. Biochim Biophys Acta 2013;1832:2414-24.

4 Krenz M, Robbins J. Impact of beta-myosin heavy chain expression on cardiac function during stress. J Am Coll Cardiol 2004;44:2390-7. 